\title{
Lattice Kinetic Modeling of the Anisotropic Growth of Two- Dimensional Islands on Barite (001) Surface
}

\author{
Santiago de Antonio Gómez, ${ }^{\dagger}$ Carlos M. Pina, ${ }^{*,+, \neq}$ and Ignacio Martin-Bragado ${ }^{*, \S}$ \\ ${ }^{\dagger}$ Department of Crystallography and Mineralogy, Complutense University of Madrid, Spain \\ ${ }^{\ddagger}$ Instituto de Geociencias IGEO, UCM-CSIC, c/José Antonio Novais 2, Madrid E-28040, Spain ${ }^{\S}$ \\ IMDEA Materials Institute, c/Eric Kandel 2, 28906 Getafe, Madrid, Spain
}
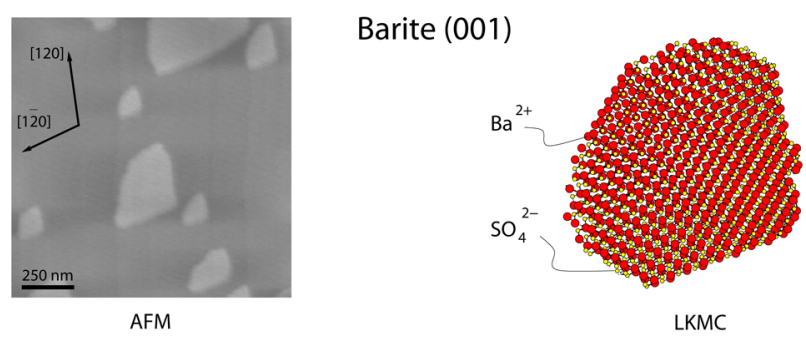

ABSTRACT: In this paper, we present simulations of twodimensional islands on the barite (001) face. These simulations were performed with MMonCa, a computer code based on the kinetic Monte Carlo technique. Our results are in excellent agreement with previous in situ atomic force microscopy (AFM) observations. Indeed, MMonCa is able to precisely reproduce both the thickness and the characteristic fan shape of barite (001) two-dimensional islands, which is defined by two straight steps parallel to the $\langle 120\rangle$ directions and a curved step connecting them. In addition, MMonCa also

simulates the orientation reversal of islands in successive growth monolayers. Fundamental for the adequate reproduction of the shape of barite (001) islands is the introduction of an anisotropy factor for the incorporation of growth units into crystallographically nonequivalent step edges. The results presented in this paper demonstrate that the consideration of simple crystallographic and geometrical constraints can be enough to provide a consistent explanation for the development of complex nanotopographies during the growth of crystals.

\section{INTRODUCTION}

Two-dimensional nucleation is the first mechanism proposed to explain the growth of atomically flat crystal faces. ${ }^{1-3}$ After the pioneering ideas of Kossel, Volmer, and Stranski, a number of physical models have been developed to predict the growth kinetics of crystal faces by means of the formation of twodimensional islands. Among them, the so-called birth and spread model provides one of the most realistic physical pictures of crystal surfaces during their growth. ${ }^{4}$ This model is based on the following main assumptions: (i) above a critical super-saturation, two-dimensional islands nucleate randomly on crystal surfaces at a measurable rate; (ii) on a given crystal face, these islands spread laterally at velocities that are independent of the island size and of the crystallographic direction (i.e., the spreading is isotropic and disk-shaped islands are formed); (iii) the coalescence of islands during their growth leads to the formation of a crystal monolayer; (iv) the nucleation of new islands can occur even before the monolayer underneath is completed; and (v) the repeated nucleation and spreading of islands result in the advancement of crystal faces. In situ atomic force microscopy (AFM) observations of inorganic and organic crystal surfaces have confirmed most of the assumptions (predictions) of the birth and spread model. ${ }^{5-11}$ Only the isotropic spreading of the twodimensional islands seems to be an unusual feature. Instead, islands often propagate over crystal surfaces with velocities that vary with the crystallographic direction. This is evidenced by the diversity of island shapes observed on different crystal faces of different compounds or on different faces of the same compound. $6,7,12$ Furthermore, it has been demonstrated that, when the growth anisotropy of two-dimensional islands is combined with symmetry operators that rotate growth directions in successive elementary growth monolayers (i.e., screw axes perpendicular to the crystal faces), the growth kinetics of crystal faces are strongly affected. ${ }^{6,12}$ These possible effects on the growth kinetics of crystal faces are, however, not considered by the birth and spread model, which assumes the formation of disk-shaped islands. Therefore, further improvements of the birth and spread model require a better understanding of the structural and symmetrical constraints to both the shape and the propagation of twodimensional islands on crystal faces.

Two-dimensional nucleation on barite $\left(\mathrm{BaSO}_{4}\right)$ faces has been revealed as a relatively simple model example in which the combined effect of anisotropy and symmetry on the growth behavior is clearly evidenced. ${ }^{5,7}$ AFM observations have shown that the shape of two-dimensional islands on barite faces are crystallographically controlled. On barite (001) face, twodimensional islands are half a unit cell in height and they have a characteristic fan shape. In addition, the orientation of these islands alternates in successive growth monolayers as a result of the operation of a 2-fold screw axis perpendicular to the barite (001) face. These features can only be partially explained by the 
orientation and strength of the $\mathrm{Ba}-\mathrm{SO}_{4}$ periodic bond chains (PBCs) within elementary growth layers and by comparing attachment energies of ions at different kink sites. ${ }^{6,13,14}$ However, the controlling factors of the shape and propagation of twodimensional islands on barite faces are not yet completely understood.

In this paper, we present lattice kinetic Monte Carlo (LKMC) simulations of two-dimensional nucleation on the barite (001) face. The aim of such simulations is to reproduce the growth of barite (001) islands observed by AFM. To this end, previously defined parameters of the simulation algorithm were systematically modified. The results obtained demonstrate that LKMC modeling can be satisfactorily used to introduce both symmetry and anisotropy controlling factors for two-dimensional nucleation on the barite (001) face. This is fundamental to elucidate the molecular-scale mechanisms of barite crystallization.

\section{AFM OBSERVATIONS}

AFM observations of two-dimensional nucleation on barite (001) faces were performed using a previously reported experimental proto-col. . $^{6,7,15}$ In brief, optically clear barite crystals from León (Spain) were cleaved along their (001) faces immediately prior to be placed in the fluid cell of an AFM (Multimode, Veeco Instruments). Then, deionized water (Milli-Q Millipore; resistivity $18 \mathrm{M} \Omega \mathrm{cm}$ ) was injected into the fluid cell, the AFM parameters were adjusted and, finally, supersaturated solutions with respect to barite were passed over the $(001)$ surfaces. $\mathrm{BaSO}_{4}$ aqueous solutions were prepared from reagent grade $\mathrm{Na}_{2} \mathrm{SO}_{4}$ and $\mathrm{BaCl}_{2}$ chemicals and deionized water. Super-saturations with respect to barite were calculated using the expression

$$
\beta_{\text {barite }}=a\left(\mathrm{Ba}^{2+}\right) a\left(\mathrm{SO}_{4}{ }^{2-}\right) / K_{\text {sp , barite }}
$$

where $\mathrm{a}\left(\mathrm{Ba}^{2+}\right)$ and $\mathrm{a}\left(\mathrm{SO}_{4}{ }^{2-}\right)$ are the activities of the ions calculated with PHREEQC ${ }^{16}$ and $\mathrm{K}_{\text {sp,barite }}=10^{-9.97}$ is the solubility product of barite at $25^{\circ} \mathrm{C}$. The supersaturations of the solutions employed in this work were higher than $\beta_{\text {barite }}=12$, well above the transitional supersaturation for twodimensional nucleation on the barite (001) face. ${ }^{17,18}$ The AFM images presented in this paper were recorded in contact mode while displaying the height signal. Subsequent image treatment was performed using the software provided by Nanoscope (5.30r3sr3) and Nanotec (WSxM 2.1). ${ }^{19}$ All growth experiments were conducted at room temperature.

Just a few seconds after injecting a supersaturated solution in the fluid cell of the AFM, numerous two-dimensional islands appear on the barite (001) face used as a substrate. As has been reported previously, these islands are half a unit cell in height $(\sim 0.36 \mathrm{~nm})$, and they are bounded by two straight edges parallel to the $\langle 120\rangle$ directions and a third curved edge. ${ }^{6,7} \mathrm{~A}$ more detailed observation of the islands reveals that curved edges are quite irregular and rough at the nanoscale, indicating that they display a high number of kink positions (Figure 1).

As growth proceeds, islands coalesce and, some minutes after starting the experiment, a new generation of islands appears, even before the first monolayer is completed (Figure 2a). The shape of these islands is identical to that of the islands formed underneath. However, they are rotated $180^{\circ}$ with respect to the first islands as a result of the existence of a 2 screw axis, which relates successive elementary (001) barite growth layers. The repeated nucleation and coalescence of two-dimensional islands leads to the layer-by-layer growth of the barite (001) face (Figure $2 b)$.

\section{LATTICE KINETIC MONTE CARLO MODELING}

Two-dimensional nucleation on the barite (001) face, described in the previous section, can be simulated using the lattice kinetic Monte Carlo technique (LKMC). This technique is based on the kinetic Monte Carlo algorithm. ${ }^{20}$ Its goal is to follow the dynamic evolution of a system that might be out of equilibrium. LKMC has been

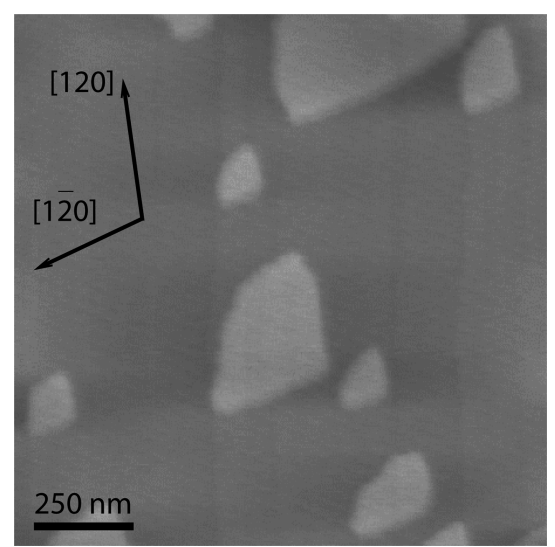

Figure 1. AFM height image of two-dimensional islands on barite (001) face growing from a supersaturated aqueous solution $\left(\beta_{\text {barite }}=26\right)$. Note the high roughness of the curved edge of the islands. The $\langle 120\rangle$ crystallographic directions are indicated by black arrows.
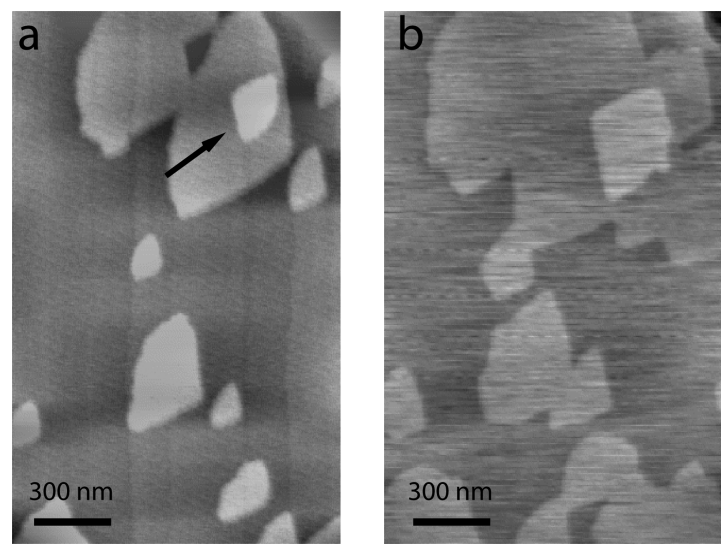

Figure 2. AFM height images showing (a) a two-dimensional island corresponding to a second nucleation event (black arrow) on barite (001) face and (b) the growth and coalescence of two-dimensional islands. Note that the orientation of the new upper island is rotated $180^{\circ}$ with respect to that of the islands formed on the previous layer.

successfully used to simulate epitaxial processes, for instance, solid phase epitaxial recrystallization ${ }^{21}$ and selective epitaxial growth. ${ }^{22}$

Barite $\left(\mathrm{BaSO}_{4}\right)$ crystallizes in the orthorhombic space group Pnma (S.G. number 62 ) with the cell parameters $a, b$, and $c$ listed in Table $1 .{ }^{14}$

Table 1. Parameters Used for $\mathrm{BaSO}_{4}$ Growth in our Lattice Kinetic Monte Carlo Simulations

\begin{tabular}{lll} 
name & \multicolumn{1}{c}{ value } & units \\
$\mathrm{a}$ & 0.88842 & $\mathrm{~nm}$ \\
$\mathrm{~b}$ & 0.54559 & $\mathrm{~nm}$ \\
$\mathrm{c}$ & 0.71569 & $\mathrm{~nm}$ \\
$\lambda$ & 0.40 & $\mathrm{~nm}$ \\
$\mathrm{e}^{-}$ & $1.61 \times 10^{-19}$ & $\mathrm{C}$ \\
$\mathrm{v}_{0}$ & $10^{-3}$ & ions/s \\
$\mathrm{Q}$ & $8.988 \times 10^{9}$ & $\mathrm{~J} \mathrm{~m} \mathrm{C}-2$ \\
$\mathrm{q}(\mathrm{Ba})$ & $+2 \mathrm{e}$ & $\mathrm{C}$ \\
$\mathrm{q}\left(\mathrm{SO} \mathrm{m}_{4}\right)$ & $-2 \mathrm{e}$ & $\mathrm{C}$ \\
$\mathrm{E}_{\mathrm{a}}$ & $-0.3 \mathrm{e}$ & $\mathrm{J}$ \\
$\mathrm{K}_{\mathrm{B}}$ & $1.38 \times 10^{-23}$ & $\mathrm{~J} / \mathrm{K}$ \\
$\mathrm{T}$ & 298 & $\mathrm{~K}$
\end{tabular}


Figure 3 shows the structure of barite projected on the (001) face in which symmetry elements are depicted. We represent the structure of

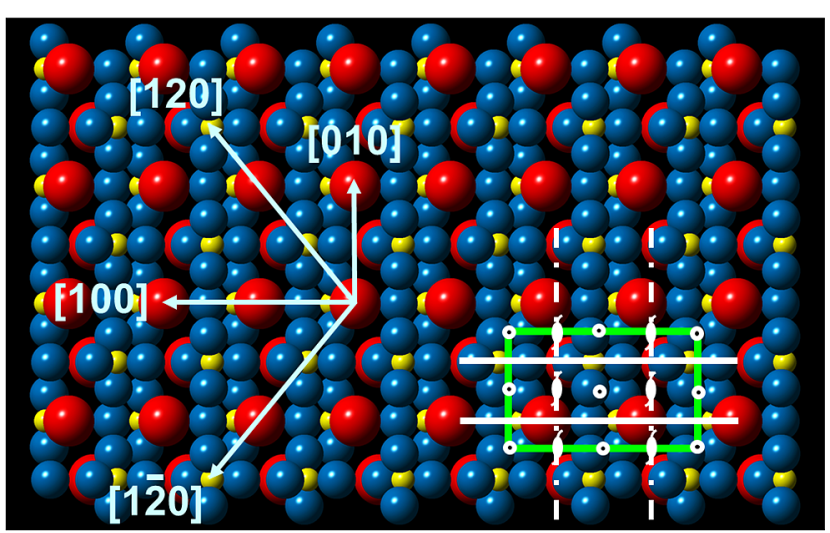

Figure 3. Barite structure projected on the (001) plane. The atoms are represented as follows: barium (red); sulfur (yellow); oxygen (blue). The rectangular surface unit cell is marked in green. Symmetry elements (mirror planes, glide planes, 2-fold screw axes, and centers of symmetry) are indicated by symbols used in the space-group descriptions of International Tables for Crystallography. ${ }^{23}$ Arrows indicate main crystallographic directions.

$\mathrm{BaSO}_{4}$ in terms of the $\mathrm{Ba}^{2+}$ and $\mathrm{SO}_{4}{ }^{2-}$ ions. The latter ion is represented as only three coordinates lying in the position of the $S$ atom, as shown in Figure 4. A rectangular simulation box is filled with

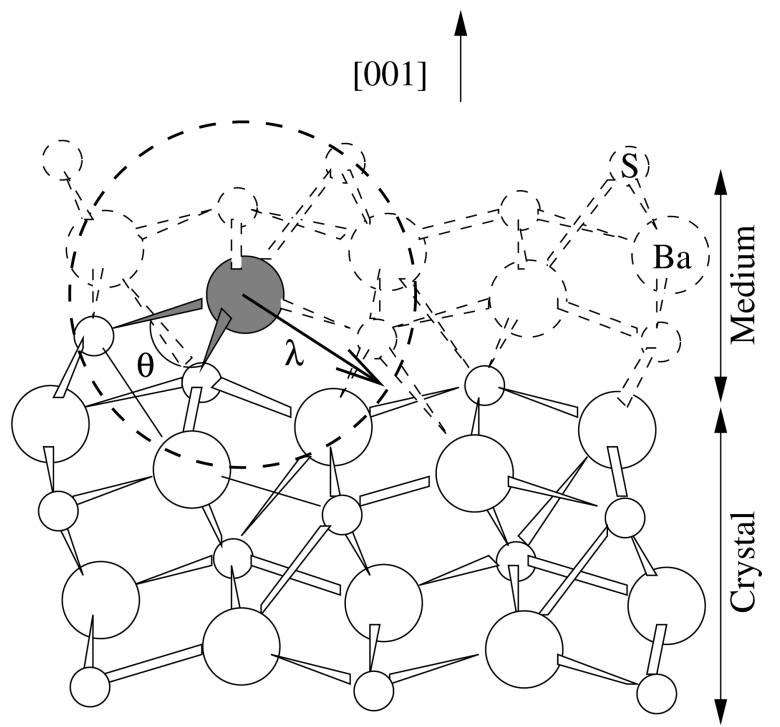

Figure 4. Two-dimensional projection of the barite structure, showing how the LKMC algorithm is applied to simulate growth. Solid white circles represent barite and dashed circles represent the "Empty" ions that will be attached by the LKMC algorithm. The solid gray atom represents a potential candidate to be incorporated into the barite structure showing its interacting neighbors.

the ions in the Wyckoff positions of the Pnma space group. A flag is assigned to each ion: "Filled" for the existing ions in the barite substrate (solid white atoms in Figure 4) and "Empty" for the potential positions of new ions to be filled during the growth process (dashed atoms in Figure 4). The LKMC algorithm assigns rates for the "Empty" positions to be transformed into "Filled", and position by position, the growth is simulated.

The transition rates, $v_{i}$, for an "Empty" i position to become "Filled" are assumed to follow an Arrhenius law:

$$
\nu_{i}=\nu_{0} \exp \left(-E_{i} /\left(K_{\mathrm{B}} T\right)\right)
$$

where $\mathrm{v}_{0}$ is a prefactor, in units of ions/s, the same for all ions, accounting for the overall growth velocity, $\mathrm{K}_{\mathrm{B}}$ is the Boltzmann constant, and $\mathrm{T}$ is the absolute temperature. For ions on the surface,

the activation energy of each ion, $E_{i}$, is computed assuming classical Coulomb's interactions:

$$
E_{i}=Q \sum_{j}^{n} q_{j} q_{i} / r_{i j}+E_{\text {aniso }}
$$

The summation extends to the $n$ "Filled" neighbors of a given ion, $Q$ being the Coulomb constant, $q_{i}$ and $q_{i}$ the charge of the involved ions, and $r_{i j}$ the distance between them. Neighbors are defined as those ions found in a capture radius $\lambda$, also shown in Figure 4 . A value of $0.40 \mathrm{~nm}$ is taken for $\lambda$ because it is the first maximum distance for which each ion has the same number of neighbors. The $\lambda$ factor has been carefully chosen to reduce the computational cost without sacrificing the accuracy of the simulation. This factor modifies the final results by changing the number of cations influencing each anion and vice versa. The cutoff given by $\lambda$ ensures that a sufficient but equal number of cations and anions are considered in the computation. An extra term, $E_{\text {aniso, }}$ is introduced to account for the anisotropy of ionic attachment to crystallographically different edges of the islands. This anisotropic term is computed as follows:

$$
E_{\text {aniso }}= \begin{cases}0 & \cos \theta>0 \\ E_{\mathrm{a}} & \cos \theta \leq 0\end{cases}
$$

Here $\theta$ is the angle between the two closest neighbors and the involved ion $\mathrm{i}$, as can be seen in Figure 4.

Once the rates for ionic attachment have been defined, the evolution of the system is produced by selecting "Empty" ions randomly but with probabilities proportional to their transition rates and increasing the simulated time involved in such step by

$$
\Delta t=-\frac{\log (s)}{R_{N}}
$$

where $R_{N}=\sum_{i}^{N} v_{i}, N$ is the total number of events to be simulated, and $s \in$ $(0,1]$ is a random number. Once the ion is chosen (gray atom in Figure 4), its flag is transformed from "Empty" to "Filled", the rates of the neighboring ions are updated, and the process continues until the requested number of ions are grown or some specified simulated time is reached. Although the LKMC algorithm provides a proper description of the system evolution with time, a precise reproduction of actual growth rates would require the calibration of the input frequency parameter $v_{0}$ using experimental data. All parameters used in the simulations are shown in Table 1.

\section{RESULTS AND DISCUSSION}

Figure 5 a shows a top view of a barite (001) two-dimensional island simulated by the MMonCa code. This island is $0.358 \mathrm{~nm}$ in height (i.e., half of the barite unit cell projected on the (001) plane), and it is bounded by two straight steps parallel to the $\langle 120\rangle$ directions and a third curved and irregular step edge that connects them. This island, which contains 5887 ions, belongs to a larger barite (001) face simulation of 18345 ions on a surface of $38 \times 29$ $\mathrm{nm}^{2}$. That simulation has been created by introducing the parameters listed in Table 1. As can be immediately recognized, the simulated island is almost identical to the experimentally produced barite (001) two-dimensional islands shown in Figure 1. We found that the introduction of the anisotropy condition, $E_{a}$ in eq 4, was essential to successfully reproduce the shape of the two-dimensional islands. The anisotropy condition accounts for the fact that step edges at the two sides of a given crystal step can be topologically different. 
a)

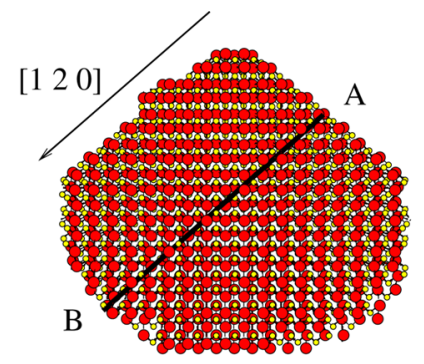

b)

B

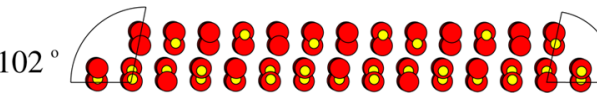

78

Figure 5. (a) Top view of barite (001) two-dimensional island. (b) Perpendicular view of the A-B section, parallel to the [120] direction. Two different step angles can be observed.

Although the relation between step edge topology and growth anisotropy is still not clear, it might be related to the enhancement of the bonding of growth units into kink sites with more open geometries. Moreover, kink sites with different geometrical constraints can influence the kinetics of attachment of growth units. The effect of such a possible anisotropy on crystal step propagation has been observed for the first time on calcite (104) surfaces. ${ }^{24-28}$ Two-dimensional islands on calcite (104) faces have a characteristic rhombus shape defined by two pairs of noncrystallographically equivalent steps (i.e., $[441]_{\mathrm{a}}$ and $[481]_{\mathrm{a}}$ and [441] $]_{0}$ and [481 $\left.]_{0}\right)$. While one of the pairs consists of steps with edges forming an acute angle of $78^{\circ}$ with the calcite (104) surface, the edges of other pair of steps form an obtuse angle of $102^{\circ}$ with such a surface. These two distinct calcite steps are usually denoted as acute and obtuse steps or, alternatively, as negative and positive steps. ${ }^{8,29-31}$ Since the incorporation of growth units into the obtuse steps is more favorable than into the acute steps, the growth of rhombus-shaped calcite (104) islands is anisotropic. This has been demonstrated by numerous AFM observations. ${ }^{8,24,25,30}$ On the barite (001) face, the scenario is similar. The two straight steps that bound the two-dimensional islands run parallel to the [120] and [120] directions (and parallel to [120] and [120] directions in the next monolayer). These steps do not contain a mirror plane or an ordinary 2-fold axis (see Figure

3). As a result, for each $\langle 120\rangle$ step, two different opposing step edges can be defined. In addition, as in the case of calcite steps, obtuse and acute $\langle 120\rangle$ steps can be distinguished. Again, the incorporation of growth units into the acute edges of the steps is less favorable than on the obtuse ones. This results in a strong growth anisotropy that controls the shape of the barite (001) islands. Figure $5 \mathrm{~b}$ shows a profile along the $\mathrm{A}-\mathrm{B}$ line in which the acute $\left(\sim 78^{\circ}\right)$ and obtuse $\left(\sim 102^{\circ}\right)$ edges of the $\langle 120\rangle$ steps can be seen. It is important to note that angles $\theta$ in eq 4 directly relate to the angles that form any step with the barite (001) plane. Thus, the ratio of obtuse to acute $\theta$ angles is higher for obtuse step angles. Therefore, the anisotropy condition expressed by eq 4 accounts for geometrical constraints of the kink positions along the crystal steps. Moreover, the anisotropy condition can be varied by changing the value given to $E_{a}$ in eq 4 . A systematic variation of the $E_{a}$ parameter allowed us to explore the effect of the anisotropy of growth on the shape of the barite (001) two-dimensional islands.
Figure 6 shows four simulations of two-dimensional islands corresponding to decreasing values of $E_{a}$. As can be seen in this
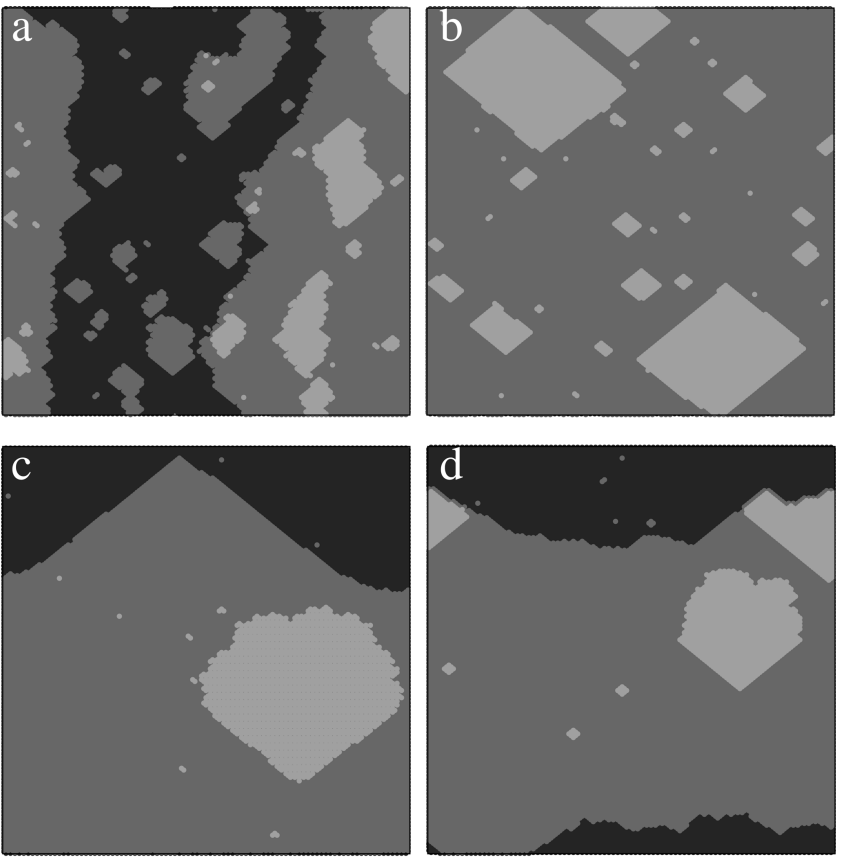

$10 \underline{\mathrm{nm}}$

Figure 6. Effect of the anisotropy parameter, $E_{a}$, on the shape of the simulated growth islands (see explanation in the main text). Scale bar in panel $d$ is valid for all simulations. $E_{a}$ values are (a) $0.30 \mathrm{eV}$, (b) $0.0 \mathrm{eV}$, (c) $-0.30 \mathrm{eV}$, and (d) $-1.5 \mathrm{eV}$.

figure, if the value of $E_{a}$ is equal to zero, simulated twodimensional islands show a rhomboid shape (Figure $6 \mathrm{~b}$ ). For negative values of $E_{a}$, the rhomboid shape vanishes and fanshaped islands, as those observed with AFM, are generated (Figure 6c). This can be explained on the basis of a simple anisotropy effect: negative $E_{a}$ values make the incorporation of growth units into obtuse step edges more favorable. As a result, two edges of the rhomboid become rougher leading to the formation of one curved edge with a fast growth rate. A further decrease of $E_{a}$ leads to an increase in the growth velocity of the curved edge, which progressively loses its characteristic curvature and becomes irregular (Figure 6d). Conversely, positive values of $E_{a}$ favor the incorporation of growth units into the acute steps edges compared with the obtuse ones. As a first morphological effect, the islands show a fir-tree-like shape elongated along the [100] direction (Figure 6a). This is a consequence of a decrease in the relative probability of attachment of growth units into the junctions between obtuse and acute step edges.

As shown by the AFM observations, the growth of barite (001) faces by the birth and spread mechanism implies the random formation and subsequent propagation of two-dimensional islands with reversed orientation in successive growth monolayers. Such a reversal is due to the existence of 2 -fold screw axes perpendicular to the barite (001) face, which relate the two $d_{002}$ elementary growth layers that constitute a barite unit cell. This feature is also simulated by the MMonCa code. Figure 7 shows a sequence of simulations in which the nucleation and spread of islands in successive growth monolayers can be seen (files with the atomic coordinates can be accessed in the Supporting Information). Two- 

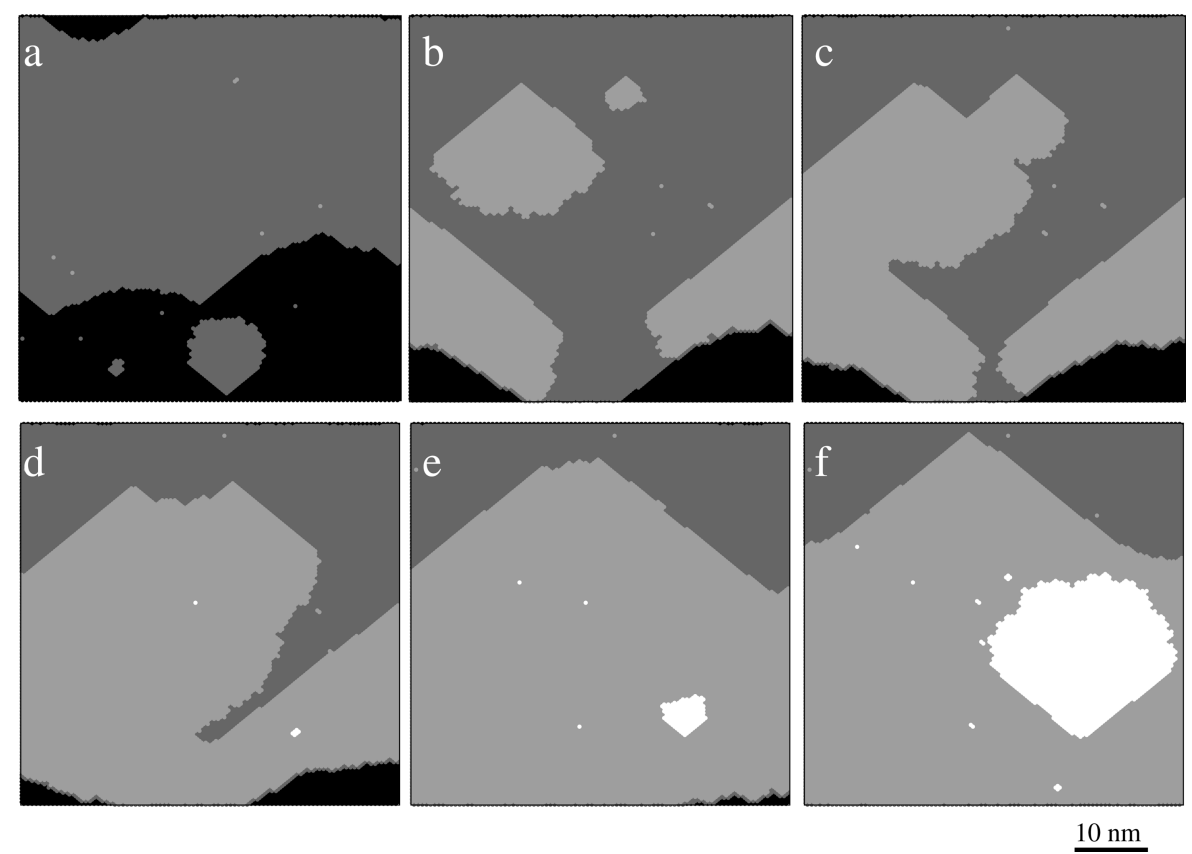

Figure 7. Top view of a growth sequence of simulated two-dimensional barite islands on barite (001). The characteristic fan-shaped islands can be observed. The excellent agreement with experimental results validates our kinetic Monte Carlo model. Scale bar in panel $\mathrm{f}$ is valid for all simulations.

dimensional islands with their characteristic fan shape appeared randomly on the barite (001) substrate (Figure 7a). The subsequent spreading of the initial islands leads to their coalescence. As a result, a first $d_{002}$ monolayer is completed. However, before this occurs, a new generation of two-dimensional islands forms on the top of the previous one (Figure $7 \mathrm{~b}, \mathrm{c})$. While the shape of the new islands is identical to the first nucleated islands, their orientation is reversed. A third generation of twodimensional islands is also formed (Figure $7 \mathrm{~d}, \mathrm{e}, \mathrm{f}$ ). Once again, a reversal of the orientation of the islands and their coalescence are observed. Therefore, the simulated growth sequence shown in Figure 7 represents a complete cycle in the growth process of the barite (001) face. Further repetitions of such a cycle will reproduce the growth of barite (001) faces by the birth and spread mechanism with all the geometrical characteristics observed by AFM.

\section{CONCLUSION}

In this paper, we have demonstrated that a simple computer code, implemented in MMonCa, based on the lattice kinetic Monte Carlo technique is able to reproduce all the features of two-dimensional nucleation observed by AFM on barite (001) faces: (i) the random formation and subsequent spreading and coalescence of twodimensional islands on the barite (001) surfaces, (ii) the $d_{002}$ thickness of the barite islands, and (iii) the fan shape of the islands and their reversed orientation in successive growth monolayers. A key issue in our MMonCa simulation is the introduction of an anisotropy factor, which allowed us to quantify the incorporation of growth units into crystallographically nonequivalent step edges. The success in simulating two-dimensional nucleation on barite (001) surfaces on the basis of simple crystallographic principles shows that the MMonCa code provides useful insights into the microscopic molecular scale of crystallization, and it is an effective tool to study the structural controls of crystal growth mechanisms at the nanoscale.

\section{ASSOCIATED CONTENT}

\section{Supporting Information}

Files of atomic positions of simulations shown in Figure 7. This material is available free of charge via the Internet at http:/l pubs.acs.org. A copy of the MMonCa simulator described in this work can be obtained by requesting an academic license through ignacio.martin@imdea.org.

\section{AUTHOR INFORMATION}

Corresponding Author

*E-mail addresses: cmpina@geo.ucm.es; ignacio.martin@ imdea.org.

Notes

The authors declare no competing financial interest.

\section{ACKNOWLEDGMENTS}

One author (I. M.-B.) wants to acknowledge funding of the project MASTIC PCIG09-GA-2011-29378 by the Marie Curie Actions Grant FP7-PEOPLE-2011-CIG program. AFM growth experiments were carried out at ICTS Centro Nacional de Microscopia Electrónica (Madrid, Spain).

\section{REFERENCES}

(1) Volmer, M. Z. Phys. 1922, 9, 193.

(2) Kossel, W. Nachr. Akad. Wiss. Göttingen, math.-phys. Kl. 1927, 135 $-143$.

(3) Stranski, I. Z. Phys. Chem. 1928, 136, 259-278.

(4) Ohara, M.; Reid, P. Modeling Crystal Growth Rates from Solution; Prentice Hall: Englewood Cliffs, N.J., 1973.

(5) Bosbach, D.; Hall, C.; Putnis, A. Chem. Geol. 1998, 151, 143160.

(6) Pina, C. M.; Becker, U.; Risthaus, P.; Bosbach, D.; Putnis, A. Nature 1998, 395, 483-486.

(7) Pina, C. M.; Bosbach, D.; Prieto, M.; Putnis, A. J. Cryst. Growth 1998, 187, 119-125.

(8) Teng, H.; Dove, P.; Yoreo, J. D. Geochim. Cosmochim. Acta 2000, 64, 2255-2266. 
(9) Astier, J.; Bokern, D.; Lapena, L.; Veesler, S. J. Cryst. Growth 2001, 226, 294-302.

(10) Malkin, A.; Kuznetsov, Y.; Yoreo, J. D.; McPherson, A. Nat. Struct. Biol. 1995, 2, 956-959.

(11) McPherson, A.; Kuznetsov, Y.; Malkin, A.; Plomp, M. J. Struct. Biol. 2003, 142, 32-46.

(12) Plomp, M.; McPherson, A.; Malkin, A. J. Cryst. Growth 2002, 237-9, 306-311.

(13) Hartman, P.; Heijnen, W. J. Cryst. Growth 1983, 63, 261-264.

(14) Hartman, P.; Strom, C. J. Cryst. Growth 1989, 97, 502-512.

(15) Sánchez-Pastor, N.; Pina, C. M.; Fernández-Díaz, L.; Astilleros, J. M. Surf. Sci. 2006, 600, 1369-1381.

(16) Parkhust, D.; Appelo, C. User's guide to PHREEQC; USGS: Reston, VA, 1999.

(17) Pina, C. M.; Enders, M.; Putnis, A. Chem. Geol. 2000, 168, 195210.

(18) Pina, C. M.; Putnis, A.; Astilleros, J. M. Chem. Geol. 2004, 204, 145-161.

(19) Horcas, I.; Fernández, R.; Gómez-Rodríguez, J. M.; Colchonero, J.; Gómez-Herrero, J.; Baró, A. M. WSxW: A software for scanning probe microscopy and a tool for nanotechnology, 2007.

(20) Bortz, A. B.; Kalos, M. H.; Lebowitz, J. L. J. Comput. Phys. 1975, $17,10-18$.
(21) Martin-Bragado, I.; Moroz, V. Appl. Phys. Lett. 2009, 95, 123123.

(22) Martin-Bragado, I. Appl. Phys. Lett. 2011, 98, 233109.

(23) Hahn, T., Ed. International Tables for Crystallography, 2nd ed.; Kluwer Academic Publishers: Dordrecht, the Netherlands, 1989; Vol. A.

(24) Hillner, P. E.; Manne, S.; Gratz, A. J.; Hansma, P. K. Ultramicroscopy 1992, 42-44, 1387-1393.

(25) Hillner, P. E.; Gratz, A. J.; Manne, S.; Hansma, P. K. Geology 1992, 20, 359-362.

(26) Grazt, A. J.; Hillner, P. E.; Hansma, P. K. Geochim. Cosmochim. Acta 1993, 57, 491-493.

(27) Paquette, A.; Reeder, R. Geochim. Cosmochim. Acta 1995, 59, 735-739.

(28) Pina, C. M.; Jordan, G. EMU Notes Mineral. 2010, 8, 229-323.

(29) Stipp, S. L. S. Geochim. Cosmochim. Acta 1999, 63, 3121-3131.

(30) Teng, H.; Dove, P.; Orme, C.; Yoreo, J. D. Science 1988, 282,

724-727.

(31) Pina, C. M.; Merkel, C.; Jordan, G. Cryst. Growth Des. 2009, 9, 4084-4090. 